\title{
Genomic diversity and antimicrobial resistance of Prevotella spp. isolated from chronic lung disease airways
}

Kasey A. Webb ${ }^{1,2}$, Olusola Olagoke ${ }^{1,2}$, Timothy Baird ${ }^{2,3}$, Jane Neill ${ }^{3}$, Amy Pham ${ }^{4}$, Timothy J. Wells ${ }^{4}$, Kay A. Ramsay ${ }^{5 \dagger}$, Scott C. Bell ${ }^{5,6,7}$, Derek S. Sarovich ${ }^{1,2^{*} \#}$, and Erin P. Price ${ }^{1,2^{*} \#}$

${ }^{1}$ GeneCology Research Centre, University of the Sunshine Coast, Sippy Downs, QLD, Australia

${ }^{2}$ Sunshine Coast Health Institute, Birtinya, QLD, Australia

${ }^{3}$ Sunshine Coast Hospital and Health Service, Sunshine Coast University Hospital, Birtinya, QLD, Australia

${ }^{4}$ University of Queensland Diamantina Institute, The University of Queensland, Woolloongabba, QLD, Australia

${ }^{5}$ QIMR Berghofer Medical Research Institute, Herston, QLD, Australia

${ }^{6}$ Adult Cystic Fibrosis Centre, The Prince Charles Hospital, Chermside, QLD, Australia

${ }^{7}$ Translational Research Institute, Woolloongabba, QLD, Australia

†Present address: Child Health Research Centre, The University of Queensland, Centre for Children's Health Research, South Brisbane, QLD, Australia

${ }^{*}$ Authors contributed equally to this work

${ }^{\#}$ Corresponding authors

University of the Sunshine Coast, Locked Bag 4, Maroochydore DC, Qld, 4558, Australia

Email: dsarovich@usc.edu.au; Ph: +61 54563748

Email: eprice@usc.edu.au; Ph: +61 754565568

Key Words: Prevotella, genomics, cystic fibrosis, chronic obstructive pulmonary disease, respiratory infections, polymicrobial infections, real-time PCR, diagnostics, sputum 
medRxiv preprint doi: https://doi.org/10.1101/2021.08.30.21262864; this version posted September 8, 2021. The copyright holder for this preprint

(which was not certified by peer review) is the author/funder, who has granted medRxiv a license to display the preprint in perpetuity.

It is made available under a CC-BY-NC-ND 4.0 International license.

\section{Data summary}

2 Thirty-five Prevotella spp. genomes generated in this study are available in the Sequence Read

3 Archive (SRA) and GenBank databases under BioProject accession PRJNA742126. 


\section{Abstract}

5 Cystic fibrosis (CF) and chronic obstructive pulmonary disease (COPD) are characterised by

6 increasingly frequent acute pulmonary exacerbations that reduce life quality and length. Human

7 airways are home to a rich polymicrobial environment, including members of the obligately anaerobic

8 genus, Prevotella. Despite their commonness, surprisingly little is known about the prevalence, role,

9 genomic diversity, and antimicrobial resistance (AMR) potential of Prevotella species/strains in

10 healthy and diseased airways. Here, we used comparative genomics to develop a real-time PCR

11 assay to permit rapid Prevotella spp. quantification from cultures and clinical specimens. Assay

12 specificity was validated across a panel of Prevotella and non-Prevotella species, followed by PCR

13 screening of CF and COPD respiratory-derived cultures. Next, 35 PCR-positive isolates were

14 subjected to whole-genome sequencing. Of eight identified species, P. histicola, P. melaninogenica,

15 P. nanceiensis, P. salivae and P. denticola overlapped between participant cohorts. Phylogenomic

16 analysis revealed considerable interhost but limited intrahost diversity, suggesting patient-specific

17 lineages in the lower airways, probably from oral cavity aspirations. Correlation of phenotypic AMR

profiles with AMR gene presence identified excellent correlation between tetQ presence and

decreased doxycycline susceptibility, and ermF presence and decreased azithromycin susceptibility

21 in this cohort. All tested Prevotella isolates were tobramycin-resistant, providing a potential selection method to improve Prevotella culture retrieval rates. Our addition of 35 airway-derived Prevotella genomes to public databases will enhance ongoing efforts to unravel the role of this diverse and enigmatic genus in both chronic respiratory diseases and healthy lungs. 


\section{Introduction}

26 Chronic lung diseases are a leading cause of global healthcare burden and early mortality ${ }^{1,2}$. Although

27 cystic fibrosis (CF) and chronic obstructive pulmonary disease (COPD) have fundamentally different aetiologies, both are characterised by impaired airway mucociliary clearance, which provides a favourable environment for microbial colonisation and persistence, and a challenging environment for pathogen eradication. Infections are the primary contributor in CF and COPD respiratory exacerbations (i.e. acute worsening of symptoms), during which inflammation and irreversible airway damage can occur ${ }^{3}$. In CF, 80-95\% of deaths are associated with acute exacerbations and chronic bacterial infections, which eventually lead to respiratory failure ${ }^{4}$. In COPD, bacterial, viral, and fungal respiratory infections trigger $\sim 70-80 \%$ exacerbation events ${ }^{5}$. Despite their crucial role in reducing quality and length of life, there remain major gaps in understanding the collective microbial drivers associated with CF and COPD pathophysiology.

37 Routine culture-based diagnosis of CF and COPD exacerbations often overlooks more fastidious or uncultivable members within the airway microbiome, leaving a gap in fully understanding the complex composition and activity of $\mathrm{CF}$ and COPD microbiomes ${ }^{6,7}$. Culture-independent techniques like metataxonomics (e.g. 16S rRNA amplicon sequencing), metagenomics, and metatranscriptomics are gaining traction as adjuncts or potentially even alternatives to culture due to their ability to identify a much broader spectrum of microbes, including uncultured taxa, resulting in better diagnosis, more informed treatment strategies, and improved clinical outcomes ${ }^{8-10}$. In addition to identifying wellcharacterised microbes associated with respiratory exacerbations, molecular methods have unveiled previously unidentified microbial communities in chronic lung disease airways, including a diverse anaerobic microbiome ${ }^{11}$.

The Prevotella genus, which comprises $\geq 51$ species $^{12,13}$, is a common and often abundant obligate anaerobe in the human body, including in CF and COPD airways ${ }^{11,14-19}$. Despite its commonness, the role of Prevotella in disease progression is incompletely understood in $\mathrm{CF}^{11}$, and even less so in COPD. Several studies investigating Prevotella spp. in airway disease, particularly CF, have yielded conflicting evidence. Some studies suggest a likely pathogenic role ${ }^{18,20}$, whereas others have shown no role ${ }^{21}$, or even a benefit when Prevotella spp. are abundant ${ }^{22,23}$. A major contributor to these equivocal findings is that culture-dependent and metaxonomic methods largely fail to identify 
54 microbes to the species and strain level ${ }^{7,13,24,25}$. As such, only a handful of Prevotella studies have

55 described potential species- or strain-specific mechanisms in airway disease pathogenesis.

56 The number of Prevotella genomes has been steadily increasing in recent years. However, in

57 comparison to more intensely studied CF and COPD airway organisms, there is a relative paucity of

58 respiratory Prevotella genomic data in public databases. Most Prevotella species contain only a single

59 representative (and many were generated from a single metagenomic study) ${ }^{26}$, leading to issues with

60 correct species assignments from metagenome-assembled genomes. Further, most genomic studies

61 have concentrated on known pathogens in oral (e.g. P. intermedia and P. nigrescens), gut (e.g. $P$.

62 copri), and urogenital (e.g. P. bivia) microbiomes ${ }^{27-29}$. There is a lack of knowledge about the

63 Prevotella spp. commonly found in airways, particularly in COPD. Insufficient resolution to the species

64 and strain level has limited our understanding of Prevotella spp. diversity, function, evolution, and

65 antimicrobial resistance (AMR) potential within CF and COPD airways during exacerbations and

66 stability $^{13,24}$.

67 Here, we first used a comparative genomics approach to design and optimise a novel real-time PCR

68 assay to permit the rapid, cost-effective, and specific detection of Prevotella spp. from both culture

69 and clinical specimens. Next, 43 CF-COPD sputa and bronchial washings were cultured to isolate

70 individual Prevotella strains for whole-genome sequencing (WGS) characterisation, followed by

71 genomic analyses to determine species identity, strain relatedness, and within-host evolution.

72 Cultured isolates were subject to AMR testing across 11 clinically-relevant antibiotics, and correlated

73 with AMR determinants identified by WGS. Finally, Prevotella morphological characteristics were

74 assessed for their diagnostic value. 


\section{Methods}

Ethics statement. Collection and analysis of clinical samples was approved by The Prince Charles Hospital (TPCH) Human Research Ethics Committee (HREC), project IDs HREC/13/QPCH/127 (CF samples) and HREC/2019/QPCH/48013 (COPD samples). Site-specific approvals were obtained for CF recruitment at TPCH, Brisbane, Australia, and COPD recruitment at Sunshine Coast Hospital and Health Service, Sunshine Coast, Australia. All participants provided written consent.

Sample collection and storage. A total of $36 \mathrm{CF}$ sputa were obtained from 21 expectorating adults with CF presenting at TPCH Adult CF Centre between 2017 and 2019. Seven participants provided multiple sequential sputa (Table S1). Sputa were stored non-anaerobically at $-80^{\circ} \mathrm{C}$ in Luria-Bertani broth containing $20 \%$ glycerol, and stored for $2-24$ months prior to microbiological assessment. A total of 32 COPD sputa ( $n=21$ ) or bronchial washings (BWs; $n=11$ ) were obtained from 21 current/former tobacco smokers during community or in-hospital consult at Sunshine Coast Hospital and Health Service in 2020 (Table S1). Five participants provided multiple sequential sputa (Table S1). BWs were taken from participants with suspected lung cancer, with most collected as paired specimens from both the tumour site (biopsied after BW collection) and approximately the same position in the non-tumour contralateral (contra) lung. BWs were pelleted at $4^{\circ} \mathrm{C}$ and $\sim 9,300 \times \mathrm{g}$ for $15 \mathrm{~min}$, with supernatant removed and pellet resuspended in $800 \mu \mathrm{L}$ phosphate-buffered saline. COPD samples were stored within 12-36 hours of collection using the same parameters as described for the CF specimens, and microbiologically processed within $\sim 2$ weeks of collection. Clinical and antibiotic treatment regimens were recorded (Table S1) to permit correlation with Prevotella AMR profiles.

Microbiological preparation and storage. Clinical samples were directly subcultured onto anaerobic blood medium containing nalidixic acid and vancomycin (Thermo Fisher Scientific, Seventeen Mile Rocks, QLD, Australia; cat. no. PP2065). Plates were incubated anaerobically at $37^{\circ} \mathrm{C}$ in an AnaeroPack 2.5L jar with an AnaeroGen 2.5L gas pack (Thermo Fisher) for 2-5 days. Isolates were subcultured based on different morphotypes, with a second subculture for purity onto non-selective anaerobic blood medium (Thermo Fisher; cat. no. PP2039). Prevotella real-time PCR-positive isolates were again sub-cultured onto non-selective anaerobic blood medium for purity and stored nonanaerobically at $-80^{\circ} \mathrm{C}$ in $1 \mathrm{~mL}$ Luria-Bertani broth containing $20 \%$ glycerol. Additionally, WGSconfirmed, representative Prevotella isolates derived from CF samples were cultured from glycerol stocks onto both polyvitaminic-supplemented chocolate agar (PVCA; Edwards Group, Murrarie, QLD, 
105 Australia; cat. no. 1065) and non-selective anaerobic blood medium to assess phenotypic differences 106 over a 10-day period.

107 DNA extractions. DNA of presumptive Prevotella spp. isolates was initially extracted using $5 \%$ chelex$108100^{30}$ (Bio-Rad, Gladesville, NSW, Australia), heat-treated at $95^{\circ} \mathrm{C}$ for $20 \mathrm{~min}$, and diluted to 1:50 with molecular-grade $\mathrm{H}_{2} \mathrm{O}$ prior to PCR. DNA for WGS was extracted using the Gram-positive bacteria protocol of the DNeasy Blood and Tissue Kit (Qiagen, Chadstone, VIC, Australia). Qiagen extractions were quality-checked and quantified using the NanoDrop 2000 spectrophotometer (Thermo Fisher). Clinical specimens were extracted using 3 parts TRI Reagent LS (Sigma-Aldrich, North Ryde, NSW, Australia) to 1 part specimen, $30 \mu \mathrm{L} 0.1 \mathrm{M} 2$-mercaptoethanol, and $\sim 100 \mu \mathrm{L}$ equal mixture of 0.1 and 0.5mm zirconia beads (Daintree Scientific, St Helens, TAS, Australia). Homogenisation comprised eight rounds of $30 \mathrm{sec}$ bead beating (each round followed by $30-60 \mathrm{sec}$ on wet ice) using the 'medium' setting of the Minilys tissue homogeniser (Bertin Instruments, Montigny-le-Bretonneux, France). After removal of the RNA aqueous phase, back extraction was performed on the interphase and proteinaceous phase ${ }^{31}$ to isolate total DNA.

Prevotella spp. real-time PCR assay design. Due to high levels of genetic variability among Prevotella spp., the highly conserved 16S-23S rRNA genes were targeted. A custom BLAST ${ }^{32}$ 2.10.2+ database was created using 60 Prevotella spp. genomes encompassing 26 assigned and nine unassigned species (Table S2). Discontiguous MegaBLAST was carried out against the 4.8kb P. intermedia ATCC 25611 23S-16S rRNA locus (GenBank accession CP019300.1, coordinates 63001-67800) using the custom BLAST database. BLAST .xml outputs were aligned and visualised using Geneious Prime vR10. A highly conserved area within the Prevotella spp. 16S rRNA gene was assessed further to identify potential PCR oligos, with conserved regions possessing several single nucleotide polymorphisms (SNPs) in other microbes exploited during probe and primer design. Primers and probes were initially assessed using Primer Express v3.0 (Applied Biosystems) to test melting temperature $\left(T_{\mathrm{m}}\right)$ suitability in Black Hole Quencher format (primer $T_{\mathrm{m}}=60^{\circ} \mathrm{C}$; probe $T_{\mathrm{m}}=70^{\circ} \mathrm{C}$ ). All candidate primers and probes were assessed for self- and hetero-dimer formation using Beacon Designer (http://www.premierbiosoft.com/qpcr/), with oligo sequences requiring a $\Delta G$ value of $>-4$ $\mathrm{kcal} / \mathrm{mol}$. Candidate oligos were subsequently subjected to NCBI Microbial Nucleotide BLAST and Nucleotide BLAST searches to confirm specificity for Prevotella spp.. As the $16 \mathrm{~S}$ region is highly conserved in all bacteria, the forward primer incorporated a penultimate mismatch to prevent non- 
specific binding to closely related non-Prevotella taxa. Additionally, a BHQplus probe (CAL Fluor Gold 136 540-pdCApdUApdUpdCGApdCGGGGpgUGG-BHQ1; Biosearch Technologies, Petaluma, California,

137 USA) was included to enhance specificity and to permit assay use on clinical specimens. Forward (5'-

138 TTCTCCAGtCCAGGATGTGtC-3') and reverse (5'-CGGGGATAACAGGCTGATCC-3') primers were

139 manufactured by Macrogen Inc. (Geumcheon-gu, Seoul, South Korea), with lowercase nucleotides

140 representing intentional mismatches.

141 Real-time PCRs were carried out on a Bio-Rad CFX96 thermocycler using $0.35 \mu \mathrm{M}$ primers and probe,

142 1X Sso Advanced Universal mastermix (Bio-Rad), molecular-grade $\mathrm{H} 2 \mathrm{O}$, and $1 \mu \mathrm{L}$ template, to a $5 \mu \mathrm{L}$

143 final volume. Thermocycling parameters were: $95^{\circ} \mathrm{C}$ for $2 \mathrm{~min}$, followed by 45 cycles of $95^{\circ} \mathrm{C}$ for $5 \mathrm{sec}$

144 and $60^{\circ} \mathrm{C}$ for $20 \mathrm{sec}$. DNA integrity for all clinical samples was assessed via amplification towards $\beta$ -

145 globin $^{33}$ (human) and universal $16 \mathrm{~S} \mathrm{rRNA}^{34}$ (bacteria); the universal 16S rRNA assay was also used

146 to verify bacterial culture DNA integrity, and a panfungal assay (O. Olagoke and E. Price,

147 unpublished) was used to verify Candida spp. DNA integrity.

148 Real-time PCR assay optimisation and specificity testing. The final optimised real-time PCR assay was assessed for accuracy by running against a diversity panel of six Prevotella spp. (one each of $P$. copri, P. denticola, P. histicola, P. melaninogenica, P. nigrescens, and P. salivae), 1 human DNA sample, and 40 non-Prevotella spp., with a focus on common respiratory tract microbes ( $n=1$ unless otherwise stated): Achromobacter xylosoxidans, Bordetella bronchiseptica, Burkholderia cepacia,

153 Burkholderia territorii, Burkholderia thailandensis, Candida albicans, Candida dubliniensis, Cupriavidus metallidurans, Enterobacter aerogenes, Enterobacter cloacae ( $n=3)$, Enterococcus raffinosus, Klebsiella oxytoca, Klebsiella pneumoniae ( $n=2)$, Lacticaseibacillus paracasei $(n=2)$, Pseudomonas aeruginosa ( $n=12$ ), Staphylococcus aureus ( $n=3)$, Staphylococcus haemolyticus,

157 Stenotrophomonas maltophilia, Veillonella atypica $(n=3)$, Veillonella parvula, and Veilonella dispar. All DNA was normalised spectrophotometrically to $2 \mathrm{ng} / \mu \mathrm{L}$ prior to $\mathrm{PCR}$.

Limits of detection (LoD) and quantification (LoQ) for the optimised assay were determined using $P$. melaninogenica LMG 28911 DNA, using serial dilutions of $4 \mathrm{ng} / \mu \mathrm{L}$ to $4 \times 10^{-6} \mathrm{ng} / \mu \mathrm{L}$ across 8 replicates per dilution. The upper and lower LoD/LoQ limits were determined as described elsewhere ${ }^{35}$. Genome equivalence was determined based on an average Prevotella genome size of 3.29Mbp and a single copy of the 16S rRNA gene. 
164 16S rRNA gene diversity profiling (metataxonomics). Metataxonomics was performed by the

165 Australian Genome Research Facility (AGRF; Melbourne, VIC, Australia) for total CF DNA and ID

166 Genomics (Seattle, WA, USA) for total COPD DNA using standard protocols for V3-V4 (i.e. 341F and 167 806R) primers.

168 Antimicrobial resistance (AMR) profiling. Twenty-eight glycerol-stocked Prevotella cultures were 169 subcultured onto non-selective anaerobic blood medium, or PVCA where no growth was obtained on 170 the anaerobic medium ( $n=2)$, and incubated at $37^{\circ} \mathrm{C}$ under anaerobic conditions for 2-3 days. Cultures 171 were then resuspended into phosphate-buffered saline to $\mathrm{OD}_{600}=0.75$ (Biochrom, Cambridge, UK).

172 Six supplemented chocolate plates per isolate were lawn-inoculated with the OD-normalised cells as 173 previously described ${ }^{36}$, and antibiotic discs (Edwards Group) towards 11 antibiotics (amoxicillin-

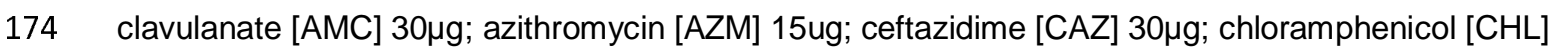

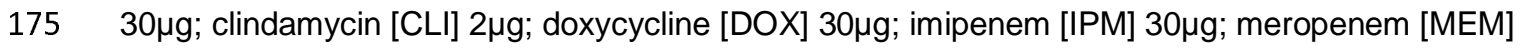

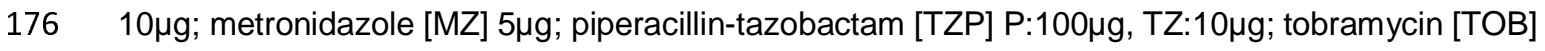
$17710 \mu \mathrm{g}$ ) were placed onto inoculated plates. Plates were incubated at $37^{\circ} \mathrm{C}$ under anaerobic conditions for $48 \mathrm{~h}$ prior to measurement. As no disc diffusion reference ranges exist for Prevotella spp., growth up to the disc (i.e. $6 \mathrm{~mm}$ diameter) was denoted as AMR, which underestimates the true rate of AMR but provides a clear cut-off for unambiguous interpretation. Diameters $\leq 30 \mathrm{~mm}$ for any antibiotic were denoted as reduced susceptibility.

182 Whole-Genome Sequencing (WGS). PCR-confirmed Prevotella isolates were sequenced using the 183 Illumina HiSeq 2500 platform with 150bp seqWell plexWell paired-end read chemistry at AGRF.

184 Genome assemblies. Prevotella genomes were de novo assembled using default Microbial Genome 185 Assembly Pipeline v1.1 parameters (https://github.com/dsarov/MGAP---Microbial-Genome-

186 Assembler-Pipeline) or SPAdes ${ }^{37}$.

187 Speciation and mixture analysis. Prevotella spp. assemblies were initially assessed for species 188 identification and mixtures using the Ribosomal $\mathrm{MLST}^{38}$ tool. Isolates containing mixtures of 189 Prevotella and non-Prevotella species $(n=8)$, or multiple Prevotella species $(n=1)$ (Table S3), were 190 excluded from further investigation.

191 Phylogenetic analysis. A custom BLAST database of Prevotella genomes ( $n=234)$ comprising taxa of 192 known ( $n=56)$ and unassigned $(n=40)$ species, and including non-mixed isolate genomes sequenced 
193 in this study ( $n=35$ genomes), was created using BLAST 2.10.2+. BLAST analysis was performed 194 against the full-length 1,534bp P. melaninogenica 16S rRNA gene (RefSeq ID: NC_014371.1; bases 195 962,784-964,317). Hits were filtered to remove redundancies (final $n=199$ ), and reads aligned using 196 ClustalX2 v2.1 $1^{39}$. A dendogram was constructed using the neighbour-joining method and the Tamura-

197 Nei model and viewed in FigTree v1.4.0 (http://tree.bio.ed.ac.uk/software/figtree/) using midpoint 198 rooting.

199 Phylogenomics. Variant identification of our CF-COPD genomes ('SCHI' prefix) was performed using 200 SPANDx v $4.0^{40}$. Reference genomes for P. denticola, P. histicola, P. melaninogenica, P. nanceiensis, 201 and P. salivae were downloaded from GenBank (IDs GCA_000193395.1, GCA_000234055.1, 202 GCA_000144405.1, GCA_000163055.2, and GCA_000477535.1, respectively) and used for read 203 mapping. Synthetic reads were generated from GenBank genome assemblies for $P$. denticola 204 (GCA_000191765.2,GCA_000759205.1,GCA_000421205.1,GCA_003515045.1, and 205 GCA_900454835.1), P. histicola (GCA_000613925.1), P. melaninogenica (GCA_000163035.1, 206 GCA_000759305.1, GCA_002208725.2, GCA_003609775.1, and GCF_000517825.1), P. nanceiensis (GCA_000517825.1 and GCA_000613985.1), and P. salivae (GCA_000185845.1 and GCA_902374235.1) using ART v2.3.7 $7^{41}$.

209 Illumina reads for P. histicola T05-04T (SRR1518629) and P. nanceiensis ADL-403 (SRR755359)

210 were downloaded from the Sequence Read Archive database and quality filtered using Trimmomatic $211 \mathrm{v} 0.39^{42}$ parameters described elsewhere ${ }^{43}$. The resulting biallelic, orthologous SNP matrix generated 212 by SPANDx was phylogenetically reconstructed using the maximum parsimony heuristic function of

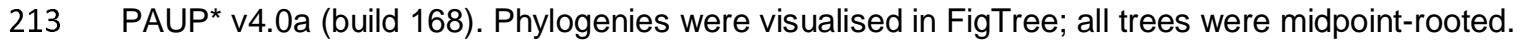

214 AMR determinant analysis. The Resistance Gene Identifier v5.1.1, which employs the Comprehensive 215 Antibiotic Resistance Database (CARD) v3.1. $0^{44}$, was used to identify putative AMR determinants. 


\section{Results and Discussion}

217 Molecular typing approaches have been instrumental in unveiling previously untapped microbial

218 diversity in both healthy and diseased lungs. Using these techniques, Prevotella spp. have consistently been identified as one of the most prevalent taxa in CF and COPD airways ${ }^{10,14,19}$. However, most studies to date have employed 16S metataxonomics (usually targeting the V3-V4 gene region), which only characterises Prevotella to the genus level, despite species and strain resolution being fundamental to unravelling the role of these anaerobes in chronic lung disease. To address this knowledge gap, our study used a combination of culture-dependent and -independent methods to examine the genomic and phenotypic diversity, and prevalence, of Prevotella spp. present in CF and COPD airways.

226 We first used a comparative genomics-guided approach to develop an accurate real-time PCR assay

227 to enable the rapid detection of Prevotella spp., to the exclusion of all other organisms, both from cultures and clinical specimens. Our PCR assay was reasonably sensitive, with LoD and LoQ both being $400 \mathrm{fg} / \mu \mathrm{L}$, or 111 genome equivalents (Figure 1). When tested against a diverse panel of 47 microbial and human DNA, the PCR demonstrated $100 \%$ specificity, amplifying all six tested Prevotella species, but none of the non-Prevotella spp. DNA and 10 no-template controls. On clinical specimens, the PCR identified Prevotella in $81 \%$ CF and 71\% COPD participants, and in $81 \%$ CF and 66\% COPD specimens (Table 1). This difference in detection rates between diseases was expected given the generally lower total bacterial load in COPD airways ${ }^{1}$. Metataxonomics had higher Prevotella sensitivity than PCR, identifying Prevotella in six PCR-negative samples, although there was one false-negative (SCHI0049.C.3, which was PCR- and culture-positive); there was otherwise near-perfect correlation between PCR and metataxonomics (Table S3). In the six PCR-negative samples, Prevotella relative abundance was very low $(0.004-0.3 \%$; median $=0.05 \%)$, indicating that DNA levels were below the PCR LoD threshold. Thus, culture-independent methods are highly suitable for accurate Prevotella spp. detection from polymicrobial specimens, particularly real-time

241 PCR, which is often performed upon clinical sample receipt, thereby permitting same-day detection of

242 Prevotella in respiratory samples.

243 Culture of sputa and BWs onto three different agars (anaerobic blood medium containing nalidixic 244 acid and vancomycin, non-selective anaerobic blood medium, and PVCA) yielded a much lower 
246 clinical specimens, respectively, were Prevotella-positive via culture, compared with $81 \%$ and $66 \%$

247 using PCR, and $84 \%$ and $91 \%$ using metataxonomics (Table 1). The reduced retrieval rate in COPD

248 vs. CF can be explained by lower relative Prevotella abundance according to metataxonomics (0.3-

$24974.3 \%$ [median=9.6\%) for CF vs. 0.002\%-36.4\% [median=0.6\%] for COPD). The lower prevalence of

250 Prevotella using culture is likely due to multiple factors, such as an inability of culture-independent

251 methods to distinguish viable from non-viable cells, nutritional fastidiousness, cells being in a viable

252 but non-culturable state, and outcompetition from faster-growing taxa ${ }^{9,19,22}$. A prior study found that

253 four different culture media were required to recover 42 Prevotella isolates from 26 samples, and only

25420 isolates were recoverable on more than one medium ${ }^{19}$. Moreover, all four media were required to

255 recover $P$. melaninogenica from all sputum samples ${ }^{19}$, suggesting that growth requirements may be

256 species- and strain-specific. In contrast to this prior work, we used aerobic collection and storage

257 conditions, which likely precluded recovery of Prevotella taxa with poor/no aerotolerance ${ }^{45}$. Consistent

258 with their fastidiousness, one P. histicola and five P. melaninogenica initially isolated in our study

259 were unable to be resuscitated from glycerol stocks upon repeated attempts, despite previously being

260 culturable. Taken together, it is probable that several Prevotella species or strains were not recovered

261 by culture in our study due to multiple factors, thereby underestimating species and strain diversity

262 within our cohorts.

263 To gain greater insight into Prevotella residing in CF and COPD airways, we performed WGS and

264 phylogenetic analysis on cultured isolates to determine species identity and strain relatedness. From

26536 CF sputa, 27 PCR-positive Prevotella colonies were retrieved, of which 18 were Prevotella spp.;

266 the remaining nine contained a mixture of Prevotella and other taxa, or a mixture of multiple

267 Prevotella spp., due to insufficient subculturing for purity prior to WGS (Table S3). To avoid known

268 bioinformatic analysis issues associated with variant calling from mixtures ${ }^{46}$, these mixed data were

269 excluded from phylogenetic analysis. Among the 18 isolates, WGS identified seven Prevotella spp.: $P$.

270 histicola ( $n=5)$, P. melaninogenica ( $n=5), P$. denticola ( $n=3), P$. nanceiensis $(n=2), P$. jejuni $(n=1), P$.

271 nigrescens $(n=1)$, and $P$. salivae $(n=1)$. Of the 17 COPD-derived isolates, none were mixed due to a

272 greater number of passages carried out to obtain purity. WGS identified six Prevotella spp.: $P$.

273 melaninogenica ( $n=8), P$. histicola $(n=3), P$. salivae $(n=3), P$. buccae $(n=1), P$. denticola $(n=1)$, and $P$.

274 nanceiensis $(n=1)$ (Table S3). Despite being fundamentally different diseases, there was little

275 difference in Prevotella species found in CF and COPD, with only $P$. jejuni and $P$. nigrescens being 
276 unique to $\mathrm{CF}$, and $P$. buccae being unique to COPD. One explanation for this overlap is that the oral

277 cavity and oropharynx are common habitats for all retrieved species, except $P$. jejuni $I^{47}$; it is thus

278 possible that some strains we isolated were upper airway residents. Whilst oral cavity contamination

279 is unavoidable during respiratory secretion collection, especially for sputum, microbes can also enter

280 lower airways via small-volume oral cavity aspirations; indeed, the risk of microbial colonisation and persistence is greatly increased in mucociliary-impaired CF and COPD airways ${ }^{24,48-52}$. In support of lower airway origin, we identified Prevotella spp. in both sputa and BWs. Unlike sputa, BWs are collected via bronchoscopy and thus far less prone to oral/oropharynx contamination, providing greater confidence that many of our retrieved Prevotella isolates originated from the lower airways.

The origin of $P$. jejuni in one CF participant is somewhat perplexing. This species was first documented in the small intestine of a child with coeliac disease in $2012^{53}$, and little is currently known about the distribution of this organism in healthy and diseased niches. One possibility is that $P$. jejuni entered the CF airways via inhalation of gastroesophageal aspirations. Alternatively, microbial inter-compartment cross-talk between the lungs and gut (the 'Gut-Lung axis') via the mesenteric lymphatic system may explain its presence in the lungs ${ }^{54}$. As a relatively new taxon, little is known about possible mechanisms of $P$. jejuni pathogenesis, with more work needed to determine the prevalence and virulence potential of this bacterium in both healthy and diseased airways.

The most frequently isolated species in our study $-P$. melaninogenica (37\%), P. histicola (23\%), $P$. salivae (11\%), P. denticola (11\%), and P. nanceiensis (9\%) - are common in $\mathrm{CF}^{14,15,19}$ and $\mathrm{COPD}^{25}$ airways. However, their precise role in airway disease is poorly understood due to conflicting reports about their beneficial vs. pathogenic potential. For instance, $P$. melaninogenica, $P$. salivae, and $P$. nanceiensis may inhibit non-typeable Haemophilus influenzae-induced pro-inflammatory cytokine production by lung dendritic cells ${ }^{52}$, increased $P$. melaninogenica prevalence in CF airways has been associated with improved lung function ${ }^{15}$, and $P$. histicola can mute pro-inflammatory pathways triggered by $P$. aeruginosa in $\mathrm{CF}$ airways ${ }^{55}$. In contrast, $P$. melaninogenica produced the greatest variety of short-chain fatty acids (associated with inflammation) amongst five tested anaerobes ${ }^{18}$, and $P$. denticola shares similarities in its lipid A moieties to $P$. intermedia (a known pathogenic species), pointing towards pro-inflammatory traits ${ }^{56}$. Finally, certain strains of the periodontal diseaseassociated species, $P$. nigrescens, a close relative of $P$. intermedia, are thought to encode an enzymatic cell hydrolysis mechanism that destroys tissues. Given the reported conflicting roles of 
306 Prevotella spp. in chronic lung disease, these collective results suggest that virulence mechanisms

307 vary among Prevotella strains, which requires further exploration ${ }^{57,58}$. Our genomic data will assist

308 these future efforts.

309 In our experience, metagenomic analysis tools can struggle with Prevotella species-level identification

310 from respiratory specimens due to a lack of accurate representative species in public databases.

311 Exemplifying this problem, few publicly available genomic data are available for $P$. nanceiensis - only

312 three were available at the time this study was undertaken, one of which is misclassified as $P$.

313 melaninogenica, and another as Prevotella sp. (Figure 3). Thus, our addition of three P. nanceiensis

314 genomes doubles the available $P$. nanceiensis data. Similarly, there are currently only a handful of

315 nonredundant $P$. jejuni genomes in public databases, and none are from lower airway specimens; our

316 addition P. jejuni SCHI0011.S.13 genome is therefore valuable for future respiratory and

317 gastroenterological studies. Taken together, our $P$. nanceiensis and $P$. jejuni genomes will help public

318 database curation efforts, and in turn, improve species identification using metagenomics.

To determine strain relatedness within and between participant airways, we undertook within-species analyses of our $P$. denticola, $P$. histicola, $P$. melaninogenica, $P$. nanceiensis and $P$. salivae genomes (Figure 3). Whole-genome SNP analysis revealed high levels of strain and interhost diversity in all examined Prevotella species. For example, genetic variation was as high as $\sim 72 \mathrm{k}$ SNPs among $P$. histicola strains, an impressive feat for an organism encoding a 3Mbp genome, and $P$. nanceiensis strains SCHI0010.S.3 and SCHI0011.S.12 were more closely related to strains isolated from France and the US, respectively, than to other strains retrieved from other Australian participants. In contrast, intrahost diversity was relatively limited (0-297 SNPs), with two instances of genetically indistinguishable strains: P. histicola SCHI0034.S.5 and SCHI0034.S.6, and P. melaninogenica SCHI0034.S.7 and SCHI0034.S.9 (Figure 3); the latter pair were retrieved from a BW and sputum collected on the same day (Table S1; Table S3). The only exception was P. melaninogenica from SCHI0027, where two genetically distinct lineages separated by $>20 \mathrm{k}$ SNPs were retrieved from this participant. There was no evidence of genetically-related strains between any of the Australian participants for any Prevotella spp. (Figure 3). These findings indicate that interhost diversity is very high, whereas within-host Prevotella diversity is limited, possibly due to frequent microaspiration of Prevotella from upper to lower airways (or via the gut-lung axis) rather than chronic infection; however, more in-depth sampling is required to investigate Prevotella persistence. 
336 Antibiotics are routinely used in the maintenance and treatment of acute respiratory infections in CF

337 and COPD ${ }^{15,59}$. Given its uncertain pathogenic role, Prevotella spp. are not targeted by these

338 treatment regimens ${ }^{60}$. Despite this, AMR rates in Prevotella are trending upwards ${ }^{59}$, possibly due to

339 bystander selection. To examine this phenomenon, we determined AMR profiles for all culturable

340 Prevotella strains ( $n=27)$ using disc diffusion against 11 antibiotics commonly used to treat lung

341 infections ${ }^{15,59}$, or which have known activity against anaerobic taxa ${ }^{47}$. AMR (AZM, CAZ, CLI, MZ,

342 TOB) or reduced susceptibility (AMC, AZM, CAZ, DOX, MEM) was observed in at least one strain,

343 whereas all were susceptible towards CHL, TZP, and IPM, an observation supported by previous

344 studies $^{47,59,61}$. Reflecting much greater lifetime antimicrobial use in the CF cohort ${ }^{62}$, the 18 tested CF

345 strains had much higher AMR incidence ( $57 \%$ vs. $8 \%$ ) and AMR gene presence ( $83 \%$ vs. $35 \%$ )

346 compared with COPD strains, and this rate increased to $100 \%$ (69\% for COPD) when including

347 strains with reduced susceptibility towards one or more antibiotics (Table 2). In addition, almost all CF

348 participants were undergoing antimicrobial treatment with at least two different antibiotic classes at

349 the time of sample collection, vs. almost no antibiotic use in the COPD participants (Table S1). One

350 strain, $\mathrm{CF}$ isolate P. histicola SCHI0009.S.6, could be classed as multidrug-resistant due to exhibiting

351 AMR towards three antibiotic classes ${ }^{63}-\beta$-lactams, lincosamides, and imidazoles - along with

352 reduced susceptibility towards the tetracycline-class antibiotic, DOX (Table 2). Our findings confirm

353 bystander AMR selection in off-target Prevotella as a direct result of heavy antimicrobial use in CF

354 (and to a much lesser extent, COPD), which may have ramifications for Prevotella persistence as a

355 potential driver of disease pathogenesis.

356 TOB was the only antibiotic tested that showed AMR in all tested isolates, including the type strain, $P$.

357 melaninogenica LMG 28911 (Table 2). This finding that concurs with prior work reporting 100\% TOB

358 resistance among 157 Prevotella isolates ${ }^{59}$. A CF microbiome study also found that Prevotella

359 abundance was largely unchanged upon TOB treatment ${ }^{64}$. These collective findings indicate that all

360 respiratory Prevotella species are intrinsically resistant to TOB. Notably, we observed that the median

361 relative Prevotella abundance according to metataxonomics was quite different between CF (9.6\%)

362 vs. COPD (0.6\%) specimens (Table S3). Although the basis of this higher relative Prevotella

363 abundance in $\mathrm{CF}$ is unknown, one possibility is that most of our CF participants were receiving TOB

364 at the time of sample collection (Table S1; Table 2), whereas no COPD participant was receiving this

365 antibiotic. The predominance of Prevotella spp. in the CF airways might thus be attributed to much 
366 more frequent TOB use in this cohort ${ }^{65-67}$. Although not assessed in this study, adding TOB to

367 selective media may improve Prevotella recovery rates from respiratory specimens by suppressing

368 growth of other taxa. After TOB, CLI resistance was most common (6/14 CF isolates), followed by

369 AZM (4/14 CF isolates), MZ (1/13 COPD and 3/14 CF isolates), and CAZ (2/14 CF isolates).

370 Decreased susceptibility towards AMC, CAZ, and DOX was common in both CF and COPD isolates;

371 however, only CF isolates exhibited decreased AZM susceptibility (6/14 isolates). These results

372 provide further evidence of bystander selection in Prevotella spp., with this effect especially noticeable

373 in CF-derived isolates.

374 AMR genes represent a major concern in infection control as these genes can rapidly transfer AMR

375

376

377

378

379

380

381

382

383

384

385

386

387

388

389

390

391

392

393

394

395

among different genera and species. To determine AMR gene potential, CARD analysis was

performed on the 35 CF-COPD Prevotella genomes and used to correlate horizontally acquired AMRconferring genes with AMR/decreased susceptibility phenotypes. Consistent with their higher AMR rates, $\mathrm{CF}$ isolates demonstrated greater prevalence of AMR-conferring genes compared with COPD (Table 2). Strikingly, 67\% CF strains encoded ermF, a horizontally-acquired gene that confers resistance towards macrolide, lincosamide, and streptogramin-class antibiotics such as AZM and $\mathrm{CLI}^{47,61,68}$. In contrast, ermF was absent in all COPD strains, a somewhat unexpected finding given that COPD participants are frequently given long-term macrolides (e.g. azithromycin, erythromycin) as prophylaxis to reduce exacerbation frequency ${ }^{69}$. None of the CF or COPD participants were receiving these antibiotics at the time of sample collection, suggesting that cross-resistance or prior CF antibiotic treatment may be driving ermF acquisition and maintenance in Prevotella. ermF presence correlated well with AZM and CLI AMR/decreased susceptibility (87\% and 75\%, respectively). ermF rates in our Australian isolates exceed those previously reported (23-68\% in UK, USA, and Italian isolates from both healthy and diseased participants ${ }^{68,70}$. This discrepancy is likely due to only respiratory disease-associated isolates being tested in our study, but may also be due to differences in AMR cut-offs between studies, in vivo antibiotic exposure levels, and potentially species or strain composition, noting that one study only identified most isolates to the genus level ${ }^{68}$.

tetQ, which confers resistance to tetracycline-class antibiotics (e.g. DOX $)^{68,70}$, was present in $54 \%$ of our strains, and more common in CF-derived strains (67\% vs. $41 \%)$. This rate is much higher than tetQ prevalence reported in UK CF $(21 \%)^{68}$ and human-derived Dutch $(30 \%)^{61}$ strains. . Our tetQharbouring strains consistently showed much smaller DOX disc diameters (12-30mm; median 22mm) 
compared with tetQ-negative strains (40-64mm; median 54mm) with $100 \%$ correlation between tetQ presence and decreased DOX susceptibility (Table 2). A prior study reported that $83 \%$ of tetQpositive isolates demonstrated intermediate or full resistance towards tetracycline ${ }^{68}$, suggesting that tet $Q$ is a reliable marker of reduced susceptibility towards tetracycline-class antibiotics. Taken together, these results confirm that tetQ presence robustly confers decreased DOX susceptibility in Prevotella spp. Although our dataset is small, we propose Prevotella spp. DOX disc diameters of $\geq 40 \mathrm{~mm}$ to denote strains lacking $t e t Q$, and $\leq 30 \mathrm{~mm}$ to denote strains that may be harbouring tetQ, with the caveat that other enigmatic determinants conferring reduced DOX susceptibility (e.g. chromosomal mutations) may be present in lieu of tetQ. These criteria also differentiate Prevotella spp. exhibiting decreased DOX susceptibility from DOX-sensitive strains.

The cfxA-type $\beta$-lactamase gene is the most clinically important AMR mechanism in Prevotella spp. ${ }^{71}$ as it confers AMR towards cephamycins ${ }^{61}$ and some other $\beta$-lactam antibiotics ${ }^{68}$, which are commonly used in respiratory infection treatment ${ }^{59}$. Concerningly, cfxA is often encoded on mobile elements, meaning that AMR can potentially be transmitted from Prevotella spp. to known pathogenic taxa in the respiratory microbiome ${ }^{72}$. We found cfXA alleles $2,3,4$, and/or 5 in $54 \%$ strains $(72 \% \mathrm{CF}$ and $35 \%$ COPD). cfxA3 and $c f x A 2$ were most common, being present in $37 \%$ and $14 \%$ strains, respectively. In comparison, a study of UK and US isolates reported much higher cfxA carriage rates (86\%), with $29 \%$ and $52 \%$ strains encoding $c f x A 3$ and $c f x A 2$, respectively. Their study also found that $c f x A 3$-encoding isolates exhibited significantly higher CAZ resistance levels ${ }^{68}$, which we also observed in our cfxA3harbouring strains (median disc diameter $=12 \mathrm{~mm}$ vs. $32 \mathrm{~mm}$ in non-cfxA3 strains) (Table 2). In cfxA3encoding strains displaying CAZ sensitivity, reduced $c f x A 3$ expression $^{73}$ may explain this phenotype, although this was not explored in our study. In addition, we did not test Prevotella susceptibility towards cephamycins so were unable to determine whether cfxA variants correlated with these $\beta$ lactam antibiotics.

MZ resistance was evident in 4/28 tested strains (3 CF; 1 COPD), despite none of the participants receiving nitroimidazoles at the time of sample collection. Other than TOB, MZ was the only antibiotic that exhibited AMR in any of the COPD strains. The nim genes, which encode for nitroimidazole reductase enzymes and have previously been associated with $\mathrm{MZ}$ resistance in $P$. baroniae ${ }^{74}$ and $P$. bivia $^{75}$, were not detected in any isolate. Therefore, the basis of MZ resistance in our isolates remains unknown. 
426 Of the eight species identified in our study, four are conventionally considered dark-pigmented $-P$.

427 denticola, P. histicola, P. melaninogenica, and P. nigrescens. Pigmented Prevotella reportedly

428 harbour a greater cache of virulence factors and are more commonly associated with infection ${ }^{47}$.

429 However, we found limited phenotypic differences within and between species on non-selective

430 anaerobic blood medium at Day 3 (Figure 4), with most presenting as convex, shiny, white colonies;

431 only P. nigrescens demonstrated tan pigmentation in the primary streak at this time point (Figure $\mathbf{4 F}$ ).

432 In contrast, a dark brown to black pigment was produced by $P$. nigrescens on PVCA by Day 3 (Figure

433 5D), but not on selective or non-selective anaerobic media, even by Day 10, with only medium-brown

434 colonies present (Figure 5B and 5C). P. histicola demonstrated tan-coloured pigmentation on both

435 non-selective anaerobic medium and PVCA, but only after five days (Figure 6), and unlike $P$.

436 nigrescens, did not develop black pigment on PVCA at Days 5 or 10. P. melaninogenica reportedly

437 produces a characteristic dark pigment that is commonly associated with the species ${ }^{76,77}$; yet, no

438 pigmentation was observed in the six P. melaninogenica isolates on any medium by Day 10. A likely

439 explanation for this phenomenon is that different strains require different media for pigment

440 production $^{78}$. Collectively, the limited pigmentation observed among Prevotella spp. suggests that

441 phenotypic characteristics alone should not be used for Prevotella genus or species identification.

442 We acknowledge several study limitations. First, small sample size (43 enrolled participants; 69

443 samples) and inclusion of only COPD and CF samples makes it difficult to generalise our results

444 across a larger cohorts. Nevertheless, the broad agreement of our findings with previous

445 literature ${ }^{10,14,19}$ demonstrates a reasonable estimation of species diversity in these cohorts. Second,

446 due to non-anaerobic sample handling and storage, non-aerotolerant Prevotella species may have

447 been rendered non-viable and thus not recoverable ${ }^{45}$. Future establishment of disc diffusion

448 guidelines in Prevotella will enable more accurate assessment of AMR phenotypes in this genus.

449 Third, due to our use of a strict AMR cut-off (i.e. $6 \mathrm{~mm}$ ), AMR rates in our Prevotella are almost

450 certainly underestimated. Fourth, we did not assess healthy airway microbiota due to difficulties

451 associated with obtaining respiratory secretions from healthy participants. Future work should

452 continue to seek strain-level resolution of Prevotella strains in CF and COPD airways, including the

453 design of species-specific assays towards commonly isolated and known pathogenic species,

454 assessment of Prevotella from healthy airways, and incorporation of higher-resolution molecular

455 technologies such as metagenomics and metatranscriptomics to gain a clearer understanding of 
medRxiv preprint doi: https://doi.org/10.1101/2021.08.30.21262864; this version posted September 8, 2021. The copyright holder for this preprint

(which was not certified by peer review) is the author/funder, who has granted medRxiv a license to display the preprint in perpetuity. It is made available under a CC-BY-NC-ND 4.0 International license.

456 Prevotella function within complex microbiomes. Co-culture experiments between Prevotella species

457 and known respiratory pathogens, such as $P$. aeruginosa, would be beneficial for elucidating the role

458 of Prevotella in potentiating or abrogating pathogen virulence. Finally, phylogeographic analysis of

459 Prevotella genomes may permit a deeper understanding of Prevotella spp. distribution and strain

460 diversity in chronic lung disease globally, and population genomic approaches will yield a better

461 understanding of Prevotella origin and capacity for airway-to-airway transmission. 
medRxiv preprint doi: https://doi.org/10.1101/2021.08.30.21262864; this version posted September 8, 2021. The copyright holder for this preprint

(which was not certified by peer review) is the author/funder, who has granted medRxiv a license to display the preprint in perpetuity.

\section{It is made available under a CC-BY-NC-ND 4.0 International license .}

\section{Acknowledgements}

463 We thank Tamieka Fraser (University of the Sunshine Coast) for laboratory assistance, Peter Timms

464 (University of the Sunshine Coast) and Laura Sherrard (Queen's University Belfast) for insightful

465 feedback on this manuscript, Tania Duarte (University of Queensland) for helpful discussions about

466 the challenges of Prevotella identification using current metagenomic pipelines, and the Belgian Co-

467 ordinated Collection of Micro-organisms (BCCM) for providing type strains. This work was funded by

468 Advance Queensland (awards AQRF13016-17RD2 and AQIRF0362018), the Wishlist Sunshine

469 Coast Hospital Foundation (award 2019-24), and the University of the Sunshine Coast. The authors

470 declare no conflicts of interest. 


\section{$471 \quad$ Figure legends}

473 Figure 1. Limits of detection (LoD) and quantification (LoQ) for the Prevotella real-time PCR assay.

474 The LoQ and LoD were both $400 \mathrm{fg} / \mu \mathrm{L}$ according to previously defined parameters ${ }^{35}$, or 111 genome 475 equivalents.

476 Figure 2. Full-length $16 \mathrm{~S}$ ribosomal RNA gene phylogenetic analysis of the 35 Prevotella strains

477 retrieved in this study (identified by 'SCHI' prefix) against 199 non-redundant public Prevotella

478 genomes comprising 56 taxa of known species and 40 taxa of unassigned species. Species clades

479 containing isolates identified from our cystic fibrosis (CF) and chronic obstructive pulmonary disease

480 (COPD) specimens are coloured.

Figure 3. Maximum parsimony within-species phylogenomic reconstruction of $P$. denticola, $P$. strains were retrieved from a single participant, coloured branch labels represent the number of SNPs separating these strains. $\mathrm{Cl}$, consistency index. Incorrectly speciated public genomes are denoted by an asterisk.

Figure 4. Three-day growth of Prevotella spp. on non-selective anaerobic blood medium. (A) $P$. melaninogenica; (B) P. histicola; (C) P. denticola; (D) P. nanceiensis; (E) P. jejuni; (F) P. nigrescens; (G) P. salivae; and (H) P. buccae.

Figure 5. Phenotype comparison of Prevotella nigrescens on non-selective anaerobic blood medium (top) and polyvitaminic-supplemented chocolate agar (bottom) at (A and D) 3 days; (B and E) 5 days; and ( $\mathrm{C}$ and $\mathrm{F}) 10$ days.

Figure 6. Phenotype comparison of Prevotella histicola on non-selective anaerobic blood medium

495 (top) and polyvitaminic-supplemented chocolate agar (bottom) at (A and C) 3 days and (B and D) 5 496 days. 
medRxiv preprint doi: https://doi.org/10.1101/2021.08.30.21262864; this version posted September 8, 2021. The copyright holder for this preprint (which was not certified by peer review) is the author/funder, who has granted medRxiv a license to display the preprint in perpetuity. It is made available under a CC-BY-NC-ND 4.0 International license.

\section{Tables}

498 Table 1. Prevotella spp. prevalence in cystic fibrosis (CF) and chronic obstructive pulmonary disease

499 (COPD) according to microbiological culture, real-time PCR, and 16S rRNA gene metataxonomics.

500

\begin{tabular}{|c|c|c|c|}
\hline \multicolumn{2}{|r|}{ Prevotella +ve } & CF & COPD \\
\hline \multirow{3}{*}{ 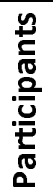 } & Culture & $11 / 21(52 \%)$ & $6 / 21(29 \%)$ \\
\hline & PCR & $17 / 21(81 \%)$ & $15 / 21(71 \%)$ \\
\hline & Metataxonomics & $15 / 17(88 \%)$ & 16/18 (89\%) \\
\hline \multirow{3}{*}{ 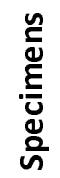 } & Culture & $16 / 36(44 \%)$ & $9 / 32$ (28\%) \\
\hline & PCR & $29 / 36(81 \%)$ & $21 / 32(66 \%)$ \\
\hline & Metataxonomics & 26/31 (84\%) & 20/22 (91\%) \\
\hline
\end{tabular}


501 Table 2. Disc diffusion and antimicrobial resistance gene results for genome-sequenced Prevotella isolates from cystic fibrosis (CF) or chronic obstructive

\begin{tabular}{|c|c|c|c|c|c|c|c|c|c|c|c|c|c|c|}
\hline \multirow{3}{*}{ Strain ID } & \multirow{3}{*}{ Prevotella sp. ${ }^{¥}$} & \multicolumn{11}{|c|}{ Antibiotic disc diffusion diameter $(\mathrm{mm})$ by antibiotic class } & \multirow{3}{*}{ cfxA allele } & \multirow{3}{*}{$\begin{array}{c}\text { Antibiotic } \\
\text { treatment }^{\ddagger}\end{array}$} \\
\hline & & \multicolumn{2}{|c|}{$\begin{array}{c}\text { Macrolide- } \\
\text { lincosamides }\end{array}$} & \multirow{2}{*}{$\begin{array}{c}\begin{array}{c}\text { Tetra- } \\
\text { cyclines }\end{array} \\
\text { DOX }\end{array}$} & \multicolumn{3}{|c|}{ Other } & \multicolumn{5}{|c|}{$\beta$-lactams } & & \\
\hline & & AZM & CLI & & CHL & MZ & тов & AMC & CAZ & TZP & IPM & MEM & & \\
\hline \multicolumn{15}{|c|}{ CF-derived isolates } \\
\hline SCHI0006.S.13 & P. histicola & 50 & 60 & $24^{*}$ & 42 & 50 & 6 & 70 & 52 & 64 & 60 & 58 & Neg & $\mathrm{Nil}$ \\
\hline SCHI0009.S.5 & P. melaninogenica & $6^{\dagger}$ & $6^{\dagger}$ & $22^{*}$ & 34 & 38 & 6 & 30 & 30 & 50 & 60 & 46 & Neg & FOF, TOB \\
\hline SCHI0009.S.6 & P. histicola & $6^{\dagger}$ & $6^{\dagger}$ & $16^{*}$ & 32 & 6 & 6 & 50 & 40 & 48 & 60 & 44 & Neg & FOF, TOB \\
\hline SCHI0010.S.3 & P. nanceiensis & $\mathrm{nt}^{\dagger}$ & $\mathrm{nt}^{\dagger}$ & $\mathrm{nt}^{*}$ & $\mathrm{nt}$ & nt & nt & $\mathrm{nt}$ & $\mathrm{nt}$ & $\mathrm{nt}$ & nt & $\mathrm{nt}$ & $c f x A 3$ & CAZ, TOB \\
\hline SCHI0011.S.12 & P. nanceiensis & 32 & 40 & $14^{*}$ & 34 & 6 & 6 & 20 & 6 & 44 & 56 & 46 & cfxA3 & CAZ, TOB \\
\hline SCHI0011.S.13 & P. jejuni & 46 & 48 & $20^{*}$ & 34 & 46 & 6 & 34 & 32 & 52 & 62 & 40 & Neg & CAZ, TOB \\
\hline SCHI0018.S.9 & P. salivae & $\mathrm{nt}^{\dagger}$ & $\mathrm{nt}^{\dagger}$ & $\mathrm{nt}^{*}$ & nt & $\mathrm{nt}$ & nt & nt & $\mathrm{nt}$ & $\mathrm{nt}$ & nt & nt & cfxA3 & $\begin{array}{c}\text { CAZ, TZP, } \\
\text { TOB }\end{array}$ \\
\hline SCHI0021.S.9 & P. denticola & $6^{\dagger}$ & $6^{\dagger}$ & $24^{*}$ & 34 & 58 & 6 & 24 & 10 & 46 & 54 & 44 & $c f x A 4$ & CAZ, TOB \\
\hline SCHI0027.S.6 & P. melaninogenica & 26 & 52 & 44 & 32 & 42 & 6 & 24 & 14 & 42 & 54 & 46 & Neg & CAZ, TOB \\
\hline SCHI0027.S.7 & P. histicola & $6^{\dagger}$ & $6^{\dagger}$ & 50 & 34 & 40 & 6 & 14 & 10 & 48 & 50 & 46 & cfxA3 & CAZ, TOB \\
\hline SCHI0027.S.9 & P. melaninogenica & $42^{\dagger}$ & $34^{\dagger}$ & 40 & 44 & 6 & 6 & 48 & 40 & 52 & 64 & 50 & $c f x A 3$ & CAZ, TOB \\
\hline SCHI0027.S.11 & P. melaninogenica & $\mathrm{nt}^{\dagger}$ & $\mathrm{nt}^{\dagger}$ & nt & nt & nt & nt & nt & nt & nt & nt & nt & $c f x A 3$ & CAZ, TOB \\
\hline SCHI0027.S.12 & P. denticola & $8^{\dagger}$ & $6^{\dagger}$ & $20^{*}$ & 34 & 44 & 6 & 10 & 6 & 38 & 46 & 38 & $c f x A 3$ & CAZ, TOB \\
\hline SCHI0027.S.13 & P. melaninogenica & $\mathrm{nt}^{\dagger}$ & $\mathrm{nt}^{\dagger}$ & $\mathrm{nt}$ & $\mathrm{nt}$ & $\mathrm{nt}$ & $\mathrm{nt}$ & $\mathrm{nt}$ & $\mathrm{nt}$ & nt & nt & $\mathrm{nt}$ & $c f \times A 3$ & CAZ, TOB \\
\hline SCHI0027.S.14 & P. denticola & $14^{\dagger}$ & $6^{\dagger}$ & 56 & 42 & 50 & 6 & 14 & 8 & 48 & 48 & 40 & $c f x A 3$ & CAZ, TOB \\
\hline SCHI0028.S.1 & P. histicola & 18 & 54 & $14^{*}$ & 38 & 40 & 6 & 50 & 32 & 44 & 60 & 26 & $c f x A 2$ & MEM, TOB \\
\hline SCHI0028.S.4 & P. histicola & 20 & 64 & $24^{*}$ & 48 & 44 & 6 & 26 & 34 & 56 & 62 & 40 & $c f x A 2$ & MEM, TOB \\
\hline SCHI0028.S.5 & $P$. nigrescens & $10^{\dagger}$ & $50^{\dagger}$ & $30^{*}$ & 50 & 60 & 6 & 36 & 24 & 50 & 56 & 50 & $c f x A 2$ & MEM, TOB \\
\hline \multicolumn{15}{|c|}{ COPD-derived isolates } \\
\hline SCHI0034.S.5 & P. histicola & 52 & 70 & 64 & 50 & 60 & 6 & 66 & 58 & 66 & 60 & 50 & $\mathrm{Neg}$ & $\mathrm{Nil}$ \\
\hline SCHI0034.S.6 & P. histicola & 44 & 60 & 58 & 42 & 50 & 6 & 58 & 50 & 58 & 58 & 50 & $\mathrm{Neg}$ & $\mathrm{Nil}$ \\
\hline SCHI0034.S.7 & P. melaninogenica & 40 & 48 & $12^{*}$ & 34 & 38 & 6 & 48 & 20 & 46 & 52 & 54 & $c f x A 3$ & $\mathrm{Nil}$ \\
\hline SCHI0034.S.8 & P. salivae & 38 & 54 & $20^{*}$ & 36 & 46 & 6 & 50 & 20 & 50 & 54 & 48 & $\mathrm{Neg}$ & $\mathrm{Nil}$ \\
\hline SCHI0034.S.9 & P. melaninogenica & $\mathrm{nt}$ & nt & $\mathrm{nt}$ & nt & nt & $\mathrm{nt}$ & $\mathrm{nt}$ & $\mathrm{nt}$ & nt & nt & nt & $c f x A 3$ & $\mathrm{Nil}$ \\
\hline SCHI0034.S.10 & P. histicola & $\mathrm{nt}$ & $\mathrm{nt}$ & $\mathrm{nt}$ & $\mathrm{nt}$ & $\mathrm{nt}$ & $\mathrm{nt}$ & $\mathrm{nt}$ & $\mathrm{nt}$ & $\mathrm{nt}$ & nt & $\mathrm{nt}$ & Neg & $\mathrm{Nil}$ \\
\hline SCHI0034.S.11 & P. salivae & 46 & 50 & $20^{*}$ & 36 & 44 & 6 & 48 & 26 & 48 & 52 & 52 & Neg & $\mathrm{Nil}$ \\
\hline SCHI0042.S.4 & P. denticola & 38 & 46 & $24^{*}$ & 36 & 46 & 6 & 18 & 14 & 44 & 50 & 42 & $c f x A 2$ & AMX \\
\hline
\end{tabular}




\begin{tabular}{|c|c|c|c|c|c|c|c|c|c|c|c|c|c|c|}
\hline & & & & & & & & & & & & & $c f x A 5$ & \\
\hline SCHI0042.S.5 & P. nanceiensis & 30 & 36 & $26^{*}$ & 34 & 6 & 6 & 14 & 12 & 36 & 52 & 52 & CfXA3 & AMX \\
\hline SCHI0043.S.1 & P. melaninogenica & $\mathrm{nt}$ & $\mathrm{nt}$ & nt & $\mathrm{nt}$ & $\mathrm{nt}$ & nt & $\mathrm{nt}$ & $\mathrm{nt}$ & $\mathrm{nt}$ & nt & $\mathrm{nt}$ & Neg & Nil \\
\hline SCHI0043.S.2 & P. melaninogenica & $\mathrm{nt}$ & $\mathrm{nt}$ & nt & nt & $\mathrm{nt}$ & $\mathrm{nt}$ & nt & $\mathrm{nt}$ & $\mathrm{nt}$ & nt & $\mathrm{nt}$ & $\mathrm{Neg}$ & Nil \\
\hline SCHI0043.S.3 & P. melaninogenica & 36 & 44 & 54 & 36 & 48 & 6 & 50 & 34 & 50 & 58 & 50 & $\mathrm{Neg}$ & Nil \\
\hline SCHI0044.S.1 & P. melaninogenica & 34 & 56 & 60 & 42 & 48 & 6 & 30 & 24 & 56 & 58 & 52 & $\mathrm{Neg}$ & $\mathrm{Nil}$ \\
\hline SCHI0047.S.2 & P. melaninogenica & 40 & 48 & $24^{*}$ & 32 & 48 & 6 & 18 & 12 & 50 & 50 & 46 & cfxA2 & Nil \\
\hline SCHI0047.S.3 & P. salivae & 40 & 48 & $24^{*}$ & 32 & 48 & 6 & 18 & 12 & 50 & 50 & 46 & $\mathrm{Neg}$ & Nil \\
\hline SCHI0047.S.4 & P. buccae & 46 & 58 & 54 & 42 & 52 & 6 & 52 & 38 & 58 & 56 & 50 & $\mathrm{Neg}$ & $\mathrm{Nil}$ \\
\hline SCHI0049.S.1 & P. melaninogenica & 44 & 58 & 58 & 44 & 50 & 6 & 24 & 20 & 58 & 60 & 52 & cfXA3 & $\mathrm{Nil}$ \\
\hline $\begin{array}{l}\text { LMG } 28911 \\
\text { (type strain) }\end{array}$ & P. melaninogenica & 40 & 54 & 50 & 36 & 46 & 6 & 50 & 50 & 54 & 52 & 38 & Unknown & Unknown \\
\hline
\end{tabular}

503 Abbreviations: AMC, amoxicillin-clavulanate; AMX, amoxicillin; AZM, azithromycin; CAZ, ceftazidime; CHL, chloramphenicol; CLI, clindamycin; DOX,

504 doxycycline; FOF, fosfomycin; IPM, imipenem; MEM, meropenem; MZ, metronidazole; Neg, negative; nt, not tested for antimicrobial sensitivity; TZP,

505 piperacillin-tazobactam; TOB, tobramycin

506 Dark grey-shaded cells represent definitive antimicrobial resistance (i.e. growth right up to the disc), light grey-shaded cells reflect reduced susceptibility

507 ( $\leq 30 \mathrm{~mm}$ diameter), and unshaded cells represent probable sensitivity (>30mm diameter)

$508{ }^{\dagger}$ Encodes ermF, which confers resistance towards erythromycin ${ }^{70}$

$509{ }^{\ddagger}$ At time of clinical specimen collection from which the isolate was derived

$510 *$ Encodes tetQ, which is associated with decreased susceptibility/resistance towards tetracycline-class antibiotics ${ }^{68,70}$ such as DOX

$511 \quad{ }^{¥}$ As determined from whole-genome sequence analysis 
513 1. Sze MA, Hogg JC, Sin DD. Bacterial microbiome of lungs in COPD. Int J Chron Obstruct Pulmon Dis $514 \quad 2014 ; 9: 229-38$.

$5152 . \quad$ Fenker DE, McDaniel CT, Panmanee W, et al. A comparison between two pathophysiologically different 516 yet microbiologically similar lung diseases: cystic fibrosis and chronic obstructive pulmonary disease. Int $J$ Respir 517 Pulm Med 2018; 5(2).

$518 \quad 3 . \quad$ Wedzicha JA, Seemungal TA. COPD exacerbations: defining their cause and prevention. Lancet 2007; 519 370(9589): 786-96.

$520 \quad 4 . \quad$ Lyczak JB, Cannon CL, Pier GB. Lung infections associated with cystic fibrosis. Clin Microbiol Rev 521 2002; 15(2): 194-222.

522 5. Jacobs DM, Pandit U, Sethi S. Acute exacerbations in chronic obstructive pulmonary disease: should 
31. Triant DA, Whitehead A. Simultaneous extraction of high-quality RNA and DNA from small tissue samples. J Hered 2009; 100(2): 246-50.

32. Altschul SF, Gish W, Miller W, Myers EW, Lipman DJ. Basic local alignment search tool. J Mol Biol 1990; 215(3): 403-10.

33. Klaassen $\mathrm{CH}$, Jeunink MA, Prinsen CF, et al. Quantification of human DNA in feces as a diagnostic test for the presence of colorectal cancer. Clin Chem 2003; 49(7): 1185-7.

34. Nadkarni MA, Martin FE, Jacques NA, Hunter N. Determination of bacterial load by real-time PCR using a broad-range (universal) probe and primers set. Microbiology (Reading) 2002; 148(Pt 1): 257-66.

35. Price EP, Dale JL, Cook JM, et al. Development and validation of Burkholderia pseudomallei-specific real-time PCR assays for clinical, environmental or forensic detection applications. PLoS One 2012; 7(5): e37723.

36. Hudzicki J. Kirby-Bauer Disk Diffusion Susceptibility Test Protocol. 2016. https://asm.org/Protocols/Kirby-Bauer-Disk-Diffusion-Susceptibility-Test-Pro (accessed 11Dec20).

37. Bankevich A, Nurk S, Antipov D, et al. SPAdes: a new genome assembly algorithm and its applications to single-cell sequencing. J Comput Biol 2012; 19(5): 455-77.

38. Jolley KA, Bliss CM, Bennett JS, et al. Ribosomal multilocus sequence typing: universal characterization of bacteria from domain to strain. Microbiology (Reading) 2012; 158(Pt 4): 1005-15.

39. Larkin MA, Blackshields G, Brown NP, et al. Clustal W and Clustal X version 2.0. Bioinformatics 2007; 23(21): 2947-8.

40. Sarovich DS, Price EP. SPANDx: a genomics pipeline for comparative analysis of large haploid whole genome re-sequencing datasets. BMC Res Notes 2014; 7: 618.

41. Huang W, Li L, Myers JR, Marth GT. ART: a next-generation sequencing read simulator. Bioinformatics 2012; 28(4): 593-4.

42. Bolger AM, Lohse M, Usadel B. Trimmomatic: a flexible trimmer for Illumina sequence data. Bioinformatics 2014; 30(15): 2114-20.

43. Price EP, Viberg LT, Kidd TJ, Bell SC, Currie BJ, Sarovich DS. Transcriptomic analysis of longitudinal Burkholderia pseudomallei infecting the cystic fibrosis lung. Microb Genom 2018; 4(8).

44. Alcock BP, Raphenya AR, Lau TTY, et al. CARD 2020: antibiotic resistome surveillance with the comprehensive antibiotic resistance database. Nucleic Acids Res 2020; 48(D1): D517-D25.

45. Silva VL, Carvalho MA, Nicoli JR, Farias LM. Aerotolerance of human clinical isolates of Prevotella spp. J Appl Microbiol 2003; 94(4): 701-7.

46. Aziz A, Currie BJ, Mayo M, Sarovich DS, Price EP. Comparative genomics confirms a rare melioidosis human-to-human transmission event and reveals incorrect phylogenomic reconstruction due to polyclonality. Microb Genom 2020; 6(2).

47. Xie $\mathrm{Y}, \mathrm{Chen} \mathrm{J}, \mathrm{He} \mathrm{J}$, et al. Antimicrobial resistance and prevalence of resistance genes of obligate anaerobes isolated from periodontal abscesses. J Periodontol 2014; 85(2): 327-34.

48. Goddard AF, Staudinger BJ, Dowd SE, et al. Direct sampling of cystic fibrosis lungs indicates that DNAbased analyses of upper-airway specimens can misrepresent lung microbiota. Proc Natl Acad Sci U S A 2012; 109(34): 13769-74.

49. Pragman AA, Lyu T, Baller JA, et al. The lung tissue microbiota of mild and moderate chronic obstructive pulmonary disease. Microbiome 2018; 6(1): 7.

50. Dickson RP, Erb-Downward JR, Freeman CM, et al. Bacterial topography of the healthy human lower respiratory tract. $m$ Bio $2017 ; 8(1)$.

51. Bassis CM, Erb-Downward JR, Dickson RP, et al. Analysis of the upper respiratory tract microbiotas as the source of the lung and gastric microbiotas in healthy individuals. mBio 2015; 6(2): e00037.

52. Larsen JM, Steen-Jensen DB, Laursen JM, et al. Divergent pro-inflammatory profile of human dendritic cells in response to commensal and pathogenic bacteria associated with the airway microbiota. PLoS One 2012; 7(2): e31976.

53. Hedberg ME, Israelsson A, Moore ERB, et al. Prevotella jejuni sp. nov., isolated from the small intestine of a child with coeliac disease. Int J Syst Evol Microbio/ 2013; 63(Pt 11): 4218-23.

54. Enaud R, Prevel R, Ciarlo E, et al. The gut-lung axis in health and respiratory diseases: a place for interorgan and inter-kingdom crosstalks. Front Cell Infect Microbiol 2020; 10: 9.

55. Bertelsen A, Elborn SJ, Schock BC. Toll like Receptor signalling by Prevotella histicola activates alternative NF-kappaB signalling in cystic fibrosis bronchial epithelial cells compared to $P$. aeruginosa. PLoS One 2020; 15(10): e0235803.

56. Di Lorenzo F, Silipo A, Matier T, et al. Prevotella denticola lipopolysaccharide from a cystic fibrosis isolate possesses a unique chemical structure. European J Org Chem 2016; 9: 1732-8.

57. Yanagisawa M, Kuriyama T, Williams DW, Nakagawa K, Karasawa T. Proteinase activity of Prevotella species associated with oral purulent infection. Curr Microbiol 2006; 52(5): 375-8.

58. Alauzet C, Marchandin H, Lozniewski A. New insights into Prevotella diversity and medical microbiology. Future Microbiol 2010; 5(11): 1695-718.

59. Sherrard LJ, Graham KA, McGrath SJ, et al. Antibiotic resistance in Prevotella species isolated from patients with cystic fibrosis. J Antimicrob Chemother 2013; 68(10): 2369-74.

60. Brill SE, Law M, El-Emir E, et al. Effects of different antibiotic classes on airway bacteria in stable COPD using culture and molecular techniques: a randomised controlled trial. Thorax 2015; 70(10): 930-8.

61. Veloo ACM, Baas WH, Haan FJ, Coco J, Rossen JW. Prevalence of antimicrobial resistance genes in Bacteroides spp. and Prevotella spp. Dutch clinical isolates. Clin Microbiol Infect 2019; 25(9): 1156 e9- e13. 
$64362 . \quad$ Sherrard LJ, Tunney MM, Elborn JS. Antimicrobial resistance in the respiratory microbiota of people with 644 cystic fibrosis. Lancet 2014; 384(9944): 703-13.

645 63. Hahn A, Burrell A, Fanous $\mathrm{H}$, et al. Antibiotic multidrug resistance in the cystic fibrosis airway

646 microbiome is associated with decreased diversity. Heliyon 2018; 4(9): e00795.

647 64. Nelson MT, Wolter DJ, Eng A, et al. Maintenance tobramycin primarily affects untargeted bacteria in the 648 CF sputum microbiome. Thorax 2020; 75(9): 780-90.

649 65. Bittar F, Richet H, Dubus JC, et al. Molecular detection of multiple emerging pathogens in sputa from 650 cystic fibrosis patients. PLoS One 2008; 3(8): e2908.

651 66. Shakil S, Khan R, Zarrilli R, Khan AU. Aminoglycosides versus bacteria--a description of the action, resistance mechanism, and nosocomial battleground. J Biomed Sci 2008; 15(1): 5-14.

67. Thornton CS, Surette MG. Potential contributions of anaerobes in cystic fibrosis airways. J Clin Microbiol 2021; 59(3).

68. Sherrard LJ, Schaible B, Graham KA, et al. Mechanisms of reduced susceptibility and genotypic prediction of antibiotic resistance in Prevotella isolated from cystic fibrosis (CF) and non-CF patients. $J$ Antimicrob Chemother 2014; 69(10): 2690-8.

69. Parameswaran GI, Sethi S. Long-term macrolide therapy in chronic obstructive pulmonary disease. CMAJ 2014; 186(15): 1148-52.

70. Arzese AR, Tomasetig L, Botta GA. Detection of tetQ and ermF antibiotic resistance genes in Prevotella and Porphyromonas isolates from clinical specimens and resident microbiota of humans. $J$ Antimicrob Chemother 2000; 45(5): 577-82.

71. Fernández-Canigia L, Cejas D, Gutkind G, Radice M. Detection and genetic characterization of betalactamases in Prevotella intermedia and Prevotella nigrescens isolated from oral cavity infections and peritonsillar abscesses. Anaerobe 2015; 33: 8-13.

72. Sherrard LJ, McGrath SJ, Mcllreavey L, et al. Production of extended-spectrum $\beta$-lactamases and the potential indirect pathogenic role of Prevotella isolates from the cystic fibrosis respiratory microbiota. Int $J$ Antimicrob Agents 2016; 47(2): 140-5.

73. Jolivet-Gougeon A, Tamanai-Shacoori Z, Desbordes L, Burggraeve N, Cormier M, Bonnaure-Mallet M. Genetic analysis of an ambler class A extended-spectrum beta-lactamase from Capnocytophaga ochracea. $J$ Clin Microbiol 2004; 42(2): 888-90.

74. $\quad$ Alauzet C, Mory F, Teyssier C, et al. Metronidazole resistance in Prevotella spp. and description of a new nim gene in Prevotella baroniae. Antimicrob Agents Chemother 2010; 54(1): 60-4.

75. Veloo ACM, Chlebowicz M, Winter HLJ, Bathoorn D, Rossen JWA. Three metronidazole-resistant Prevotella bivia strains harbour a mobile element, encoding a novel nim gene, nimK, and an efflux small MDR transporter. J Antimicrob Chemother 2018; 73(10): 2687-90.

76. Allison HE, Hillman JD. Cloning and characterization of a Prevotella melaninogenica hemolysin. Infect Immun 1997; 65(7): 2765-71.

77. Zhou X, Li Y. Subgingival microbes: from healthy microflora to disease. Atlas of Oral Microbiology: Elsevier; 2015.

78. Finegold SM. Anaerobic Gram-negative bacilli (Chapter 20). In: Baron S, ed. Medical Microbiology (4th edition). Galveston, TX: University of Texas Medical Branch at Galveston; 1996. 


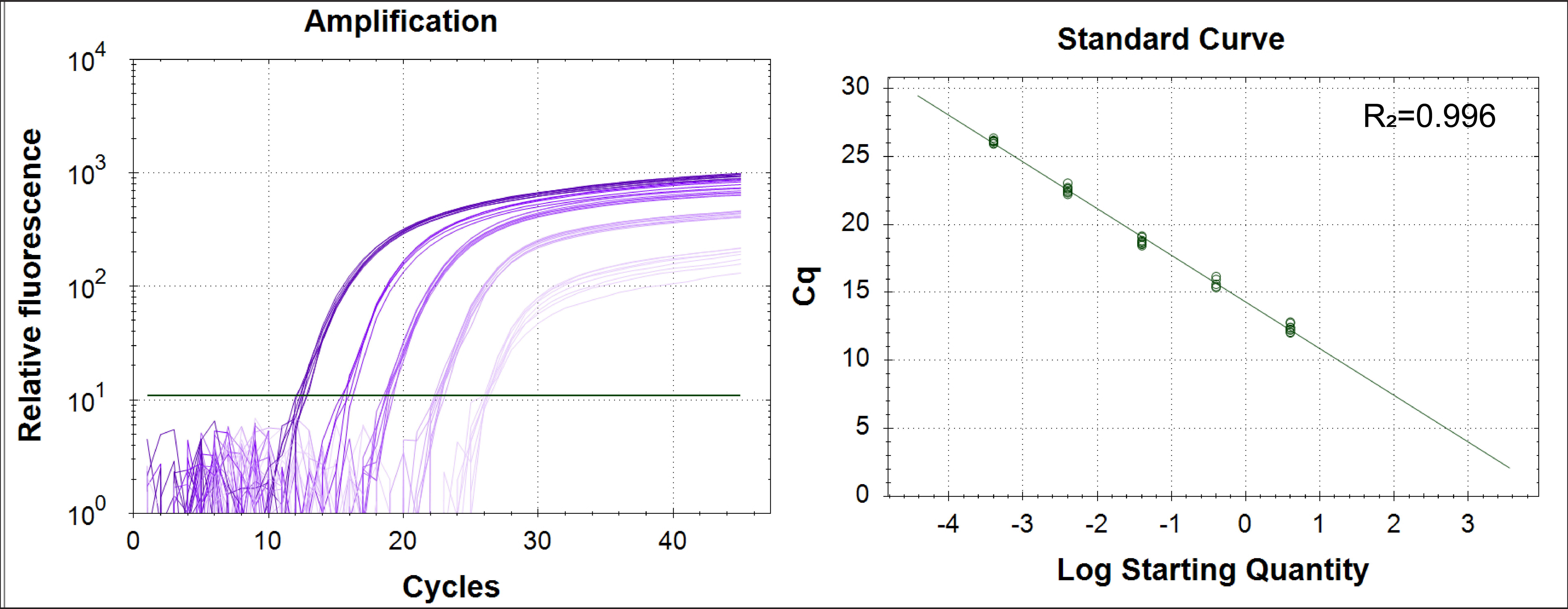




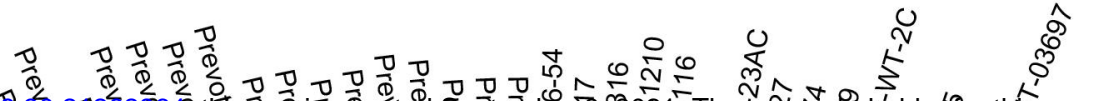

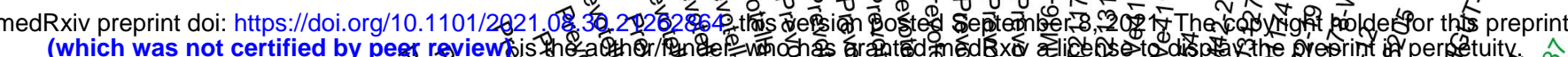

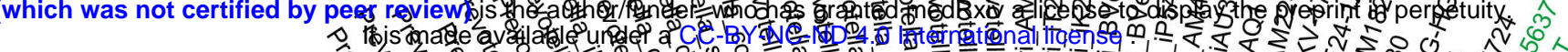

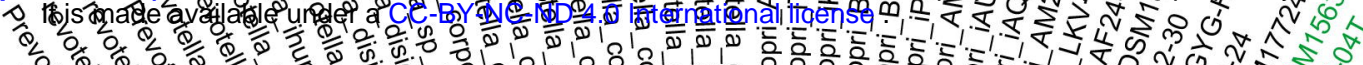

$\begin{array}{lll}0 & 0 \\ 0 & 0 & 0\end{array}$

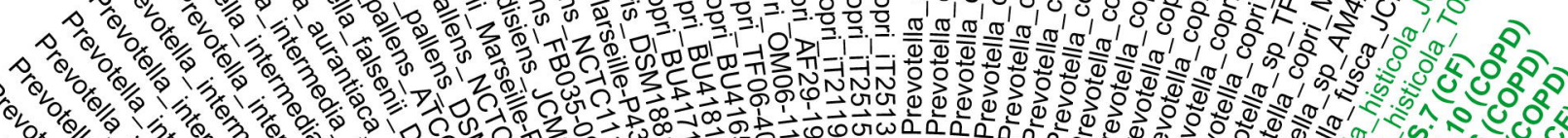

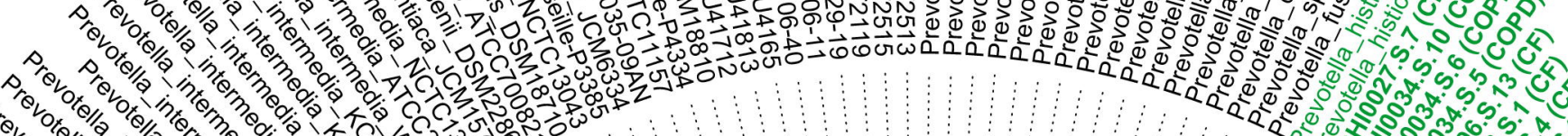

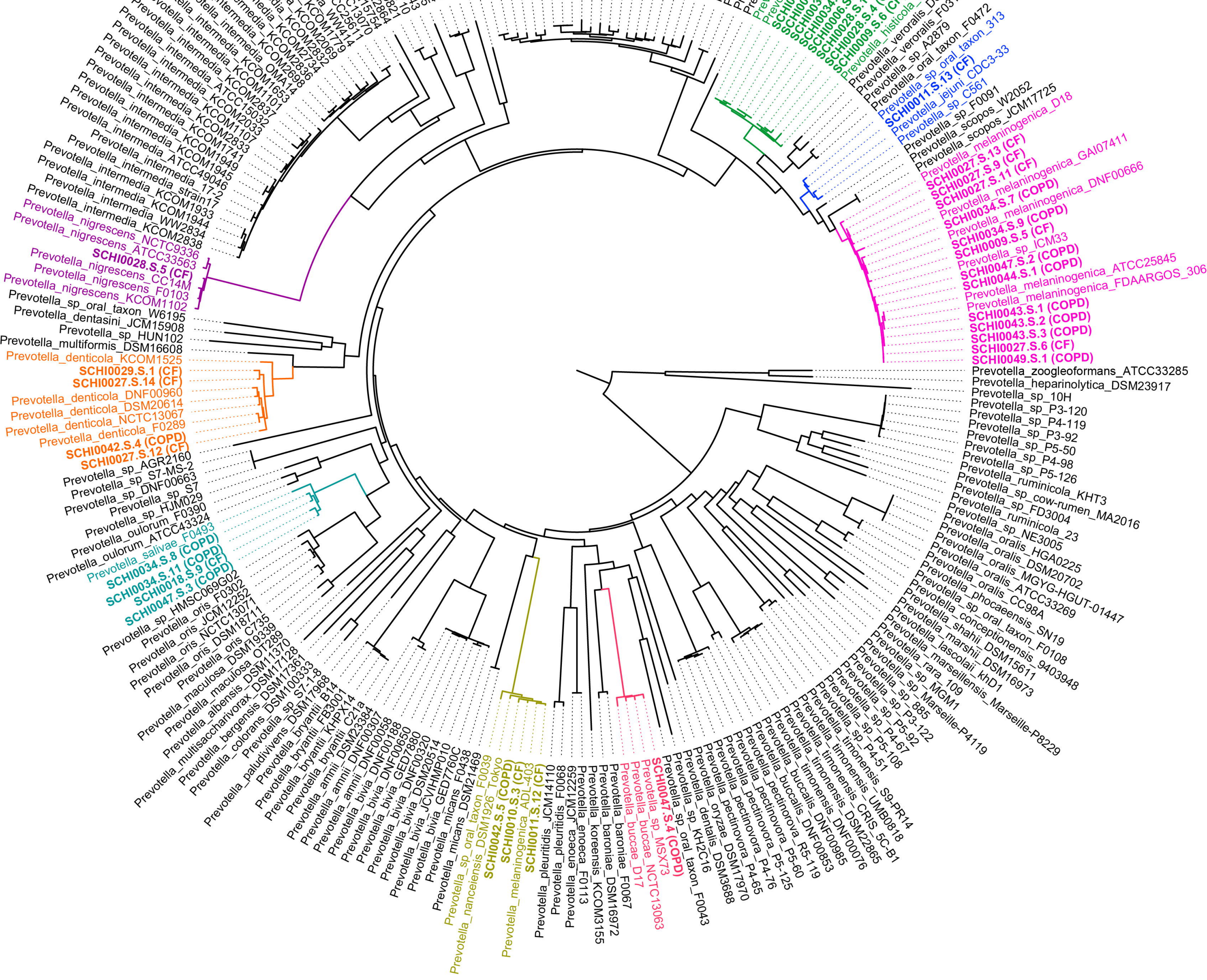




\section{Prevotella denticola}

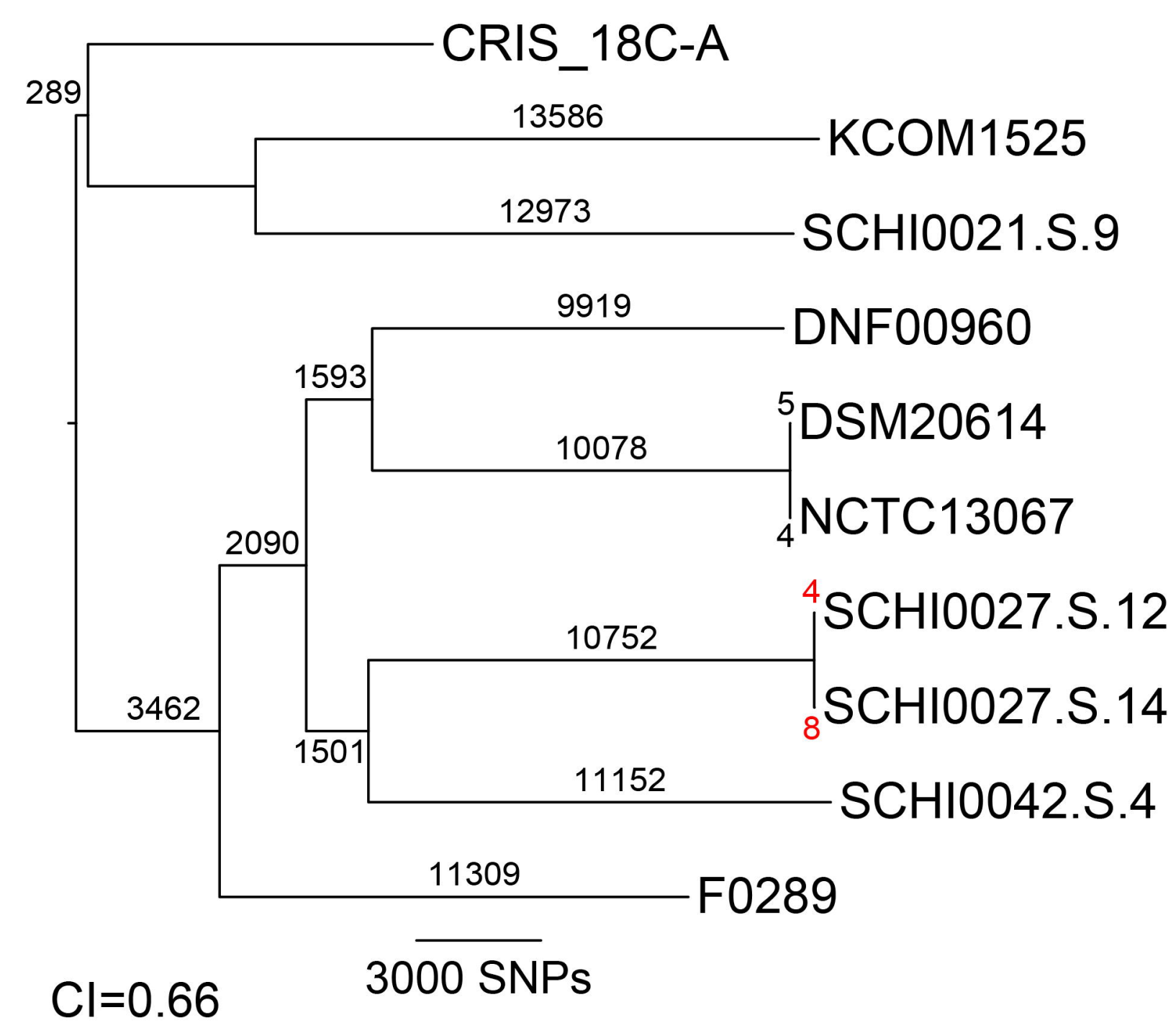

Prevotella nanceiensis

5005 Prevotella sp. oral taxon F0039*

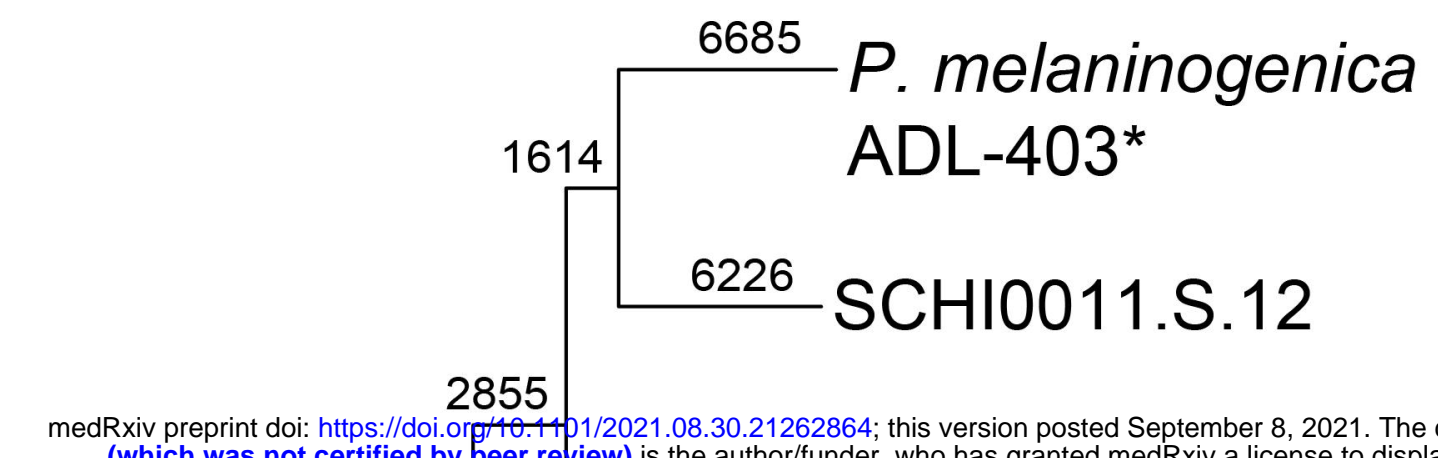

medRxiv preprint doi: https:///doi.org 1 14 1/2021.08.30.21262864; this version posted September 8, 2021. The copyright holde for this preprint
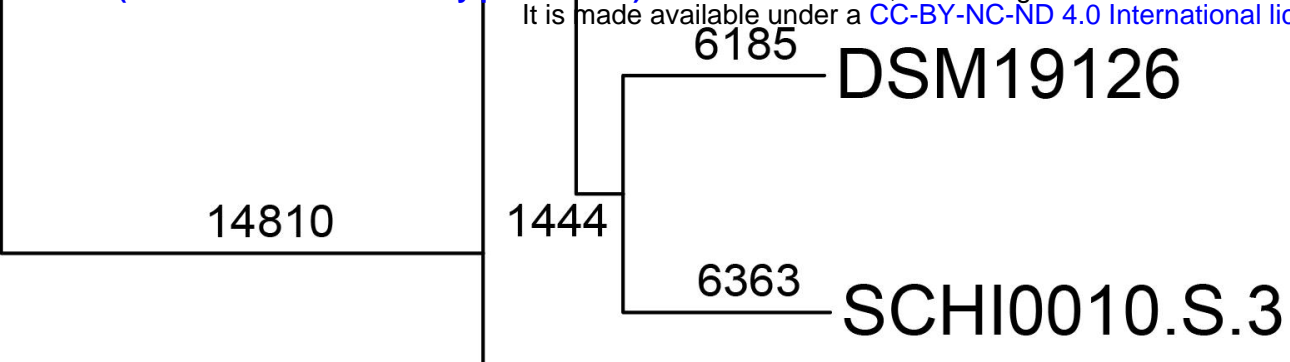

6010 SCHI0042.S.5 3000 SNPs
Prevotella histicola

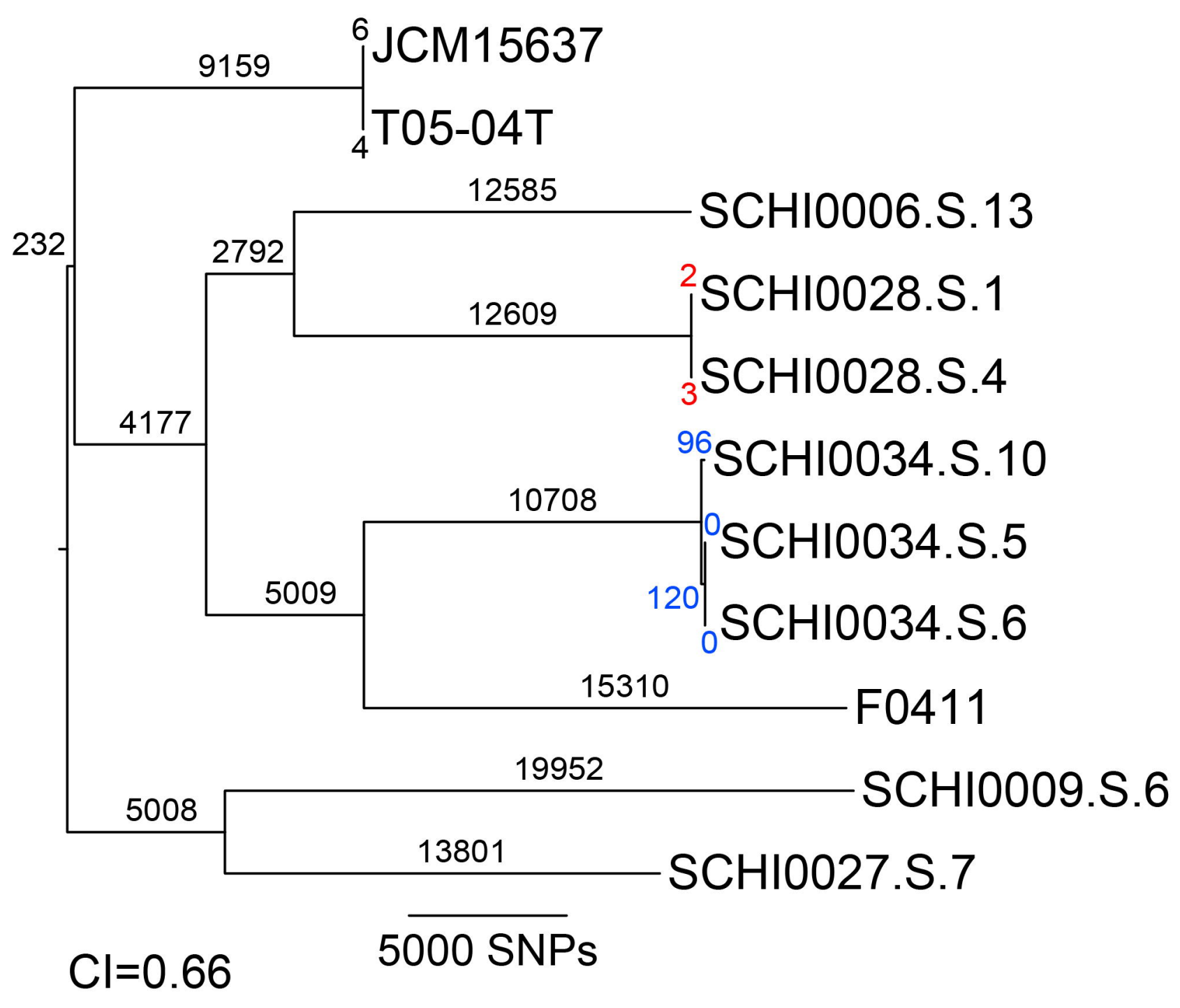




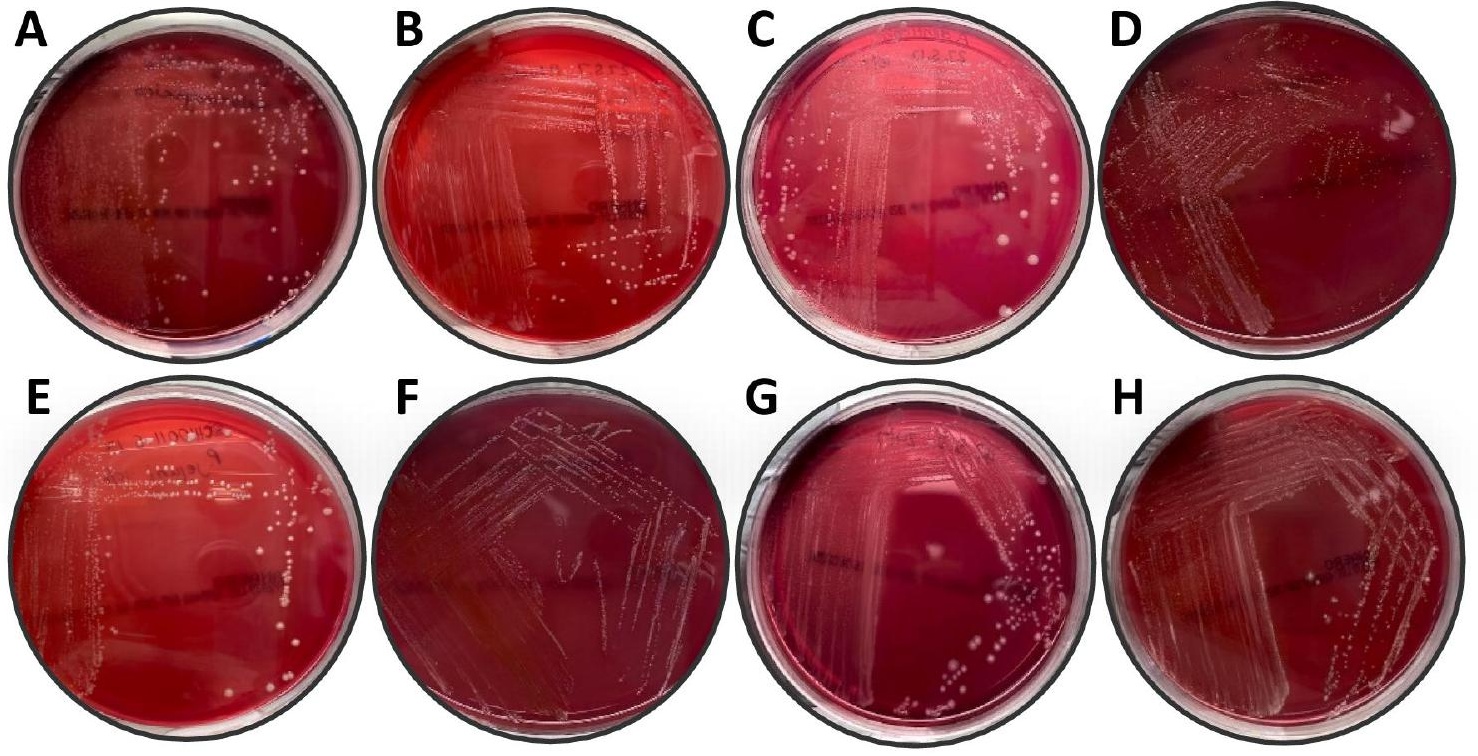




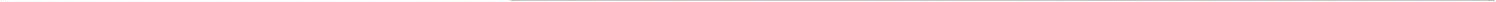




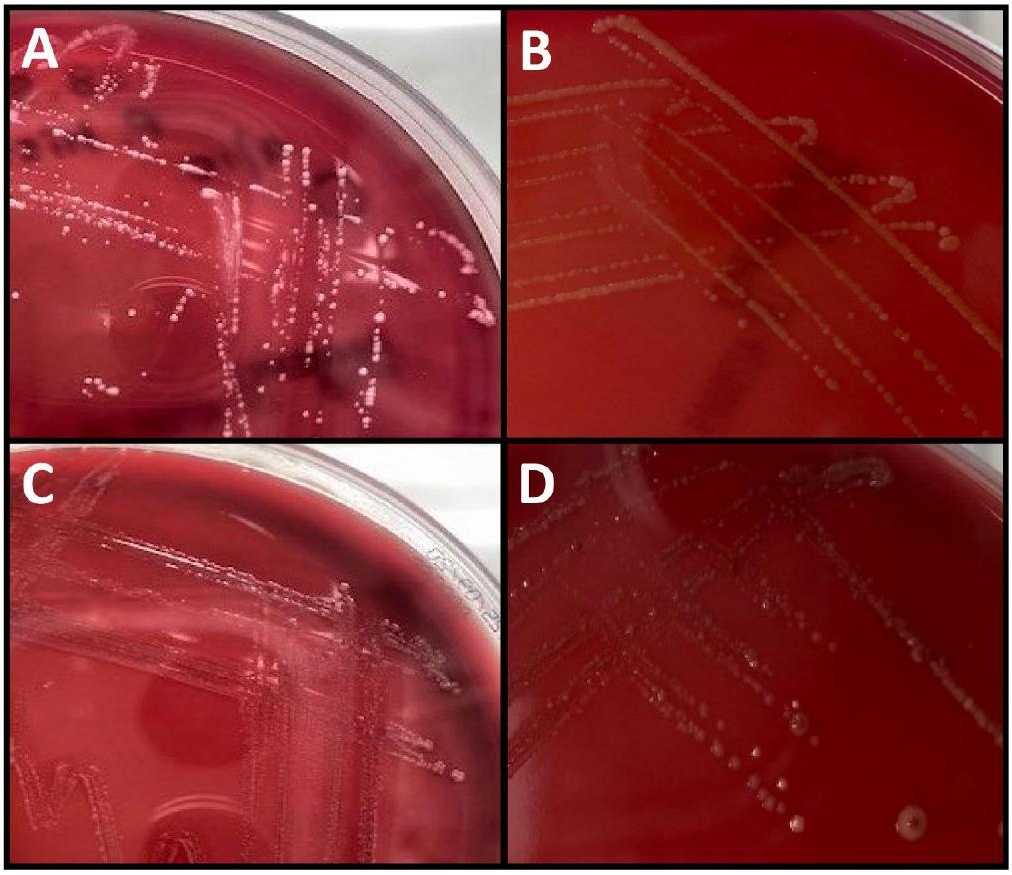

